\title{
Clinical efficacy, safety, and tolerability of fingolimod for the treatment of relapsing- remitting multiple sclerosis
}

This article was published in the following Dove Press journal:

Drug, Healthcare and Patient Safety

II December 2015

Number of times this article has been viewed

\author{
Alberto Gajofatto ${ }^{1,2}$ \\ Marco Turatti² \\ Salvatore Monaco ${ }^{1,2}$ \\ Maria Donata Benedetti ${ }^{2}$ \\ 'Department of Neurological, \\ Biomedical and Movement Sciences, \\ University of Verona, Verona, Italy; \\ ${ }^{2}$ Division of Neurology B, Verona \\ University Hospital, Verona, Italy
}

\begin{abstract}
Fingolimod is a selective immunosuppressive agent approved worldwide for the treatment of relapsing-remitting multiple sclerosis (MS), a chronic and potentially disabling neurological condition. Randomized double-blind clinical trials have shown that fingolimod significantly reduces relapse rate and ameliorates a number of brain MRI measures, including cerebral atrophy, compared to both placebo and intramuscular interferon- $\beta 1 \mathrm{a}$. The effect on disability progression remains controversial, since one Phase III trial showed a significant benefit of treatment while two others did not. Although fingolimod has a very convenient daily oral dosing, the possibility of serious cardiac, ocular, infectious, and other rare adverse events justified the decision of the European Medicines Agency to approve the drug as a second-line treatment for MS patients not responsive to first-line therapy, or those with rapidly evolving course. In the United States, fingolimod is instead authorized as a first-line treatment. The aim of this review is to describe and discuss the characteristics of fingolimod concerning its efficacy, safety, and tolerability in the clinical context of multiple sclerosis management.
\end{abstract}

Keywords: multiple sclerosis, fingolimod, safety, tolerability, efficacy

\section{Introduction}

Multiple sclerosis (MS) is a chronic demyelinating and degenerative disease of the central nervous system (CNS), characterized by recurrent episodes of neurological dysfunction, accumulation of irreversible disability, or both. The condition is associated with the pathological finding of extensive inflammation with scattered distribution in the CNS white matter, gray matter, and meninges, likely caused by an autoimmune process triggered by one or more still unidentified causal factors. ${ }^{1}$ As a consequence, no definitive or etiological treatment for MS exists, although several disease-modifying drugs (DMDs) are available that may reduce disease activity and improve the clinical course by modulating or suppressing the immune system. Currently approved DMDs in Europe, USA, and many other countries include interferon- $\beta 1 \mathrm{a}$ and $-\beta 1 \mathrm{~b}$, glatiramer acetate, mitoxantrone, natalizumab, fingolimod, teriflunomide, dimethyl fumarate, and alemtuzumab. Immunosuppressive drugs such as azathioprine, cyclophosphamide, and cladribine have a consolidated clinical use or are approved in some countries. After nearly two decades in which only injectable agents were approved for MS treatment and azathioprine was the only off-label oral option with evidence of efficacy, fingolimod (also known as FTY720) was the first DMD marketed as a single-daily capsule that showed promising therapeutic effect in MS. However, some safety issues were identified during the drug development process, after completion of trials, and in the first months of clinical use in the United States, that led to approval of fingolimod 
as a second-line DMD by the European Medicines Agency (EMA) after the US Food and Drug Administration (FDA) had licensed it as a first-line agent. In addition, contradictory results regarding efficacy on progression of disability in MS patients were found in the two pivotal Phase III trials that allowed fingolimod marketing in most countries. ${ }^{2,3}$

The aim of this review is to describe and comment on the safety, tolerability, and clinical efficacy of fingolimod for the treatment of relapsing-remitting MS.

\section{The story of an ancient Chinese fungus}

In 1855, Miles Joseph Berckley - the founder of British mycology - firstly described a fungus called Cordyceps sinclairii in its teleomorphic form (the sexual reproductive stage), ${ }^{4}$ which several years later in 1923 Curtis Gates Lloyd classified in the genus Iseria, with its anamorphic (asexual reproductive stage) name, Isaria sinclairii..$^{5}$ The fungus is endemic in southwestern China and to the alpine habitats of the Tibetan Plateau over 3,000 meters above sea level; it victimizes a particular type of cicada larvae as a host in which to propagate. The larvae usually die just beneath the soil surface, and the fungus produces white tufts, which grow up from the soil and release powdery white spores. Mushrooms species related to $I$. sinclairii have been used for centuries in Tibetan and Chinese traditional medicine as tonic, sexualenhancer, anticancer, and immune boosting drugs, which some refer to as elixir of eternal youth along with ginseng and deer antlers, although with poor supporting scientific evidence. ${ }^{6}$

In 1992, a Japanese research team headed by Dr Fujita at Kyoto University isolated from a culture broth of $I$. sinclairii a metabolite named ISP-1 or myriocin showing potent immunosuppressive properties. ${ }^{7}$ First, in vitro experiments showed that ISP-1 strongly inhibited the proliferation of T-cells in mouse allogeneic mixed lymphocyte reaction and significantly prolonged rat skin allograft survival; however, higher dose of ISP-1 induced marked toxicity in vivo. In 1995, researchers from Yoshitomi Pharmaceuticals, after several processes of simplification of ISP-1, in order to reduce its toxicity and enhance pharmacological properties, synthetized 2-amino-2-[2-(4-octylphenyl)ethyl]propane-1,3diol, a new molecule abbreviated as FTY720, giving birth to fingolimod. ${ }^{8}$ In 1997, the Japanese company sold FTY720 to Novartis Pharmaceuticals, which set up a development and research plan for fingolimod as an add-on therapy to cyclosporine after renal transplantation. However, after initial enthusiasm for the preliminary results, two large trials showed that fingolimod was less safe than mycophenolate mofetil and did not support a dose reduction of cyclosporine to prevent renal transplant rejection. ${ }^{9,10}$

At that stage, other studies had shown that FTY720 was effective in preventing experimental autoimmune encephalomyelitis (EAE; ie, the animal model of MS) and in decreasing infiltration of CD4 T-cells into the spinal cord of mice with EAE. ${ }^{11}$ The road for studies in MS patients was open. The first clinical evidence of FTY720 efficacy in MS came in 2006 with a 6-month placebo-controlled Phase II trial, followed by a 6-month extension in which all patients were switched to FTY720. ${ }^{12}$ In 2010, two major Phase III trials were published: the FTY720 Research Evaluating Effects of Daily Oral therapy in Multiple Sclerosis (FREEDOMS), a 24-month double-blind placebo-controlled study, ${ }^{2}$ and the Trial Assessing Injectable Interferon Versus FTY720 Oral in Relapsing-remitting Multiple Sclerosis (TRANSFORMS), a 12-month double-blind Phase III study comparing fingolimod and low-dose interferon- $\beta 1 \mathrm{a}$ as active treatment for MS. ${ }^{3}$ Both trials showed a significant benefit of fingolimod on relapse rate and brain MRI outcomes, which led to rapid approval of the drug with the brand name of Gilenya ${ }^{\circledR}$ (Novartis Pharmaceuticals, Basel, Switzerland) by the FDA on September 22, 2010. Just a few months later, early in 2011, the Committee for Medicinal Products for Human Use (CHMP) of EMA gave positive opinion for clinical use of Gilenya $^{\circledR}$, which in March of the same year became the first oral treatment officially approved for MS in all the European Union. In the meantime, the Cleveland Clinic 8th annual Innovation Summit had listed fingolimod at the 9th position among the top 10 medical innovations for 2011, following groundbreaking drugs for cancer and hepatitis $\mathrm{C}$ as well as technological innovations like florbetapir as a new molecular imaging biomarker for early detection of Alzheimer's disease and incisionless bariatric surgery. ${ }^{13}$ During this time, the media also helped popularize the discovery of the new "MS pill." ${ }^{14}$ But what was the buzz all about?

\section{Clinical efficacy of fingolimod in multiple sclerosis \\ Mechanism of action}

Fingolimod is a small lipophilic molecule with a sphingosinelike structure, which exerts its biological activity after phosphorylation and interaction with the sphingosine-1-phosphate (S1P) receptor family. Fingolimod shows affinity for four of the five known S1P receptors subtypes, which are abundantly expressed on the surface of a variety of cell subtypes, including but not limited to endothelial cells, lymphocytes, 
smooth muscle and cardiac myocytes, and neural cells. S1P receptor type 1 (S1PR1) is mainly expressed by lymphocytes and represents the most relevant molecular target for the therapeutic effect of fingolimod in MS. ${ }^{15}$ Fingolimod-phosphate, which is the active form of the drug, acts as a highaffinity agonist of S1PR1 causing its downregulation on the cell surface and termination of the sphingosine-dependent intracellular signaling. S1PR1 molecular pathway is essential for cell migration processes, particularly of lymphocytes subtypes expressing the "homing" receptor CCR7, such as naïve and central memory T-cells, $\mathrm{T}_{\mathrm{H}} 17$ cells, and $\mathrm{B}$-cells. S1PR1 internalization and loss of function induced by fingolimod determines an imbalance toward CCR7 signaling, which inhibits lymphocytes mobilization to the peripheral blood, leading to their segregation into secondary lymphoid organs. Importantly, effector memory T-cells do not express CCR7, and their mobilization from lymphoid tissue is not influenced by fingolimod, thus explaining why immunological surveillance is substantially preserved during treatment. Sequestration of central memory T-cells, $\mathrm{T}_{\mathrm{H}} 17$ cells, and B-cells in the peripheral lymphoid tissue significantly reduces access of autoreactive lymphocytes to the CNS of patients with MS, thus modulating the inflammatory process that starts and maintains the formation of demyelinating plaques, axonal damage, and neuronal death. Furthermore, in vitro and animal models studies have shown that fingolimod interacts with S1PR1, S1PR3, and S1PR5 expressed by neurons, astrocytes, oligodendrocytes, and microglia, promoting several neuroprotective and regenerative events, such as neural stem cell migration, neuronal injury repair, increase of endogenous brain-derived neurotrophic factor, astrocyte proliferation and migration, protection of oligodendrocytes from cell death, increase of oligodendrocytes progenitors number, and remyelination. ${ }^{16}$

\section{Evidence from clinical trials}

In 2003, fingolimod entered the clinical phase of the research pipeline for MS with the beginning of the first Phase II trial. The study enrolled 281 subjects with active relapsingremitting or secondary progressive MS who were randomized to receive daily fingolimod $1.25 \mathrm{mg}, 5 \mathrm{mg}$, or placebo for 6 months (core phase, after which patients on placebo were randomized to either fingolimod dose and the entire cohort was followed for additional 6 months). Compared to patients on placebo, subjects on both fingolimod doses had a significantly lower number of total gadolinium-enhancing lesions on monthly brain MRI up to month 6 , which was the primary outcome of the study. In addition, patients on fingolimod showed a benefit in a number of secondary outcomes, including the annualized relapse rate (ARR; $53 \%-55 \%$ relative reduction compared to placebo), proportion of patients free from relapses ( $86 \%$ fingolimod versus $66 \%$ placebo), total cumulative number of new T2 lesions, and total cumulative volume of gadolinium-enhanced lesions. No statistically significant differences were observed between placebo and fingolimod groups in neurological disability progression and brain volume change from baseline. ${ }^{12}$ Since the $5 \mathrm{mg}$ fingolimod dose showed no additional benefits and was associated with increased frequency of adverse events (AE) compared to the lower dose, subsequent Phase III trials were performed using fingolimod 1.25 and $0.5 \mathrm{mg}$ doses, which was the dosage eventually approved for clinical use. FREEDOMS was the first randomized placebo-controlled double-blind Phase III trial involving a large number of relapsing-remitting MS patients (1,272 cases, of which 1,033 completed the study) followed for 2 years. ${ }^{2}$ Subjects treated with both fingolimod doses showed a significantly reduced ARR (primary endpoint) compared to placebo, with a relative reduction of $54 \%$ and $60 \%$ for the $0.5 \mathrm{mg}$ and the $1.25 \mathrm{mg}$ doses, respectively. Compared to placebo, fingolimod 0.5 $\mathrm{mg}$ treatment also resulted in a significantly lower risk of relapse $(29.6 \%$ versus $54.4 \%)$, disability progression confirmed at 6 months (12.5\% versus 19\%), presence of new T2 lesions (49.5\% versus $78.8 \%$ ) and gadolinium-enhancing lesions $(10.3 \%$ versus $34.9 \%)$ on brain MRI at 2 years. In addition, patients receiving fingolimod had a significantly reduced brain volume loss compared to placebo over the whole study period. Data from the FREEDOMS trial were further analyzed in a major post hoc study with the objective of identifying potential patient subgroups with distinct treatment response profile. ${ }^{17}$ Overall analysis revealed that ARR reduction was consistently observed in all demographic, clinical, and MRI subgroups, with the exception of subjects over 40 years of age who had no significant ARR decrease over 2 years of fingolimod treatment compared to placebo. In addition, patients of female sex, previously treated with other DMDs, with less than 3 relapses in 2 years before study, with baseline expanded disability status scale (EDSS) score $<4.0$, or with $\mathrm{T} 2$ lesion volume $\leq 3,300 \mathrm{~mm}^{3}$ on baseline brain MRI did not have a significant benefit on the risk of disability progression at 2 years while on fingolimod compared to placebo.

TRANSFORMS was a contemporary - although shorter than FREEDOMS - double-blind randomized Phase III trial comparing the efficacy and safety of two fingolimod doses and intramuscular interferon- $\beta 1 \mathrm{a} 30 \mu \mathrm{g}$ once a week in 
1,292 relapsing-remitting MS patients followed for 1 year ( $89 \%$ of cases completed the study). ${ }^{3}$ Fingolimod treatment resulted in an ARR relative reduction up to $51 \%$ compared to interferon ( 0.20 for fingolimod $1.25 \mathrm{mg}, 0.16$ for fingolimod $0.5 \mathrm{mg}$, and 0.33 for interferon; $P<0.001$ for both fingolimod doses versus interferon) independent of previous use of other DMDs. Nearly $83 \%$ of patients on fingolimod $0.5 \mathrm{mg} /$ day remained relapse free during the trial versus $69 \%$ of patients on interferon. Overall, neurological disability progression occurred at a very low rate (less than $8 \%$ in 1 year) in the study population and no statistically significant differences were evident between fingolimod and interferon in terms of EDSS score worsening confirmed at 3 months. However, the 1-year change on the EDSS and Multiple Sclerosis Functional Composite (MSFC) favored fingolimod over interferon, although the clinical relevance of such difference is uncertain. MRI data analysis revealed that fingolimod treatment had a better outcome compared to interferon on several measures, including the number of new or enlarged brain lesions on T2-weighted images, number of gadoliniumenhancing lesions on T1-weighted images, and the change from baseline in brain volume. However, the change from baseline in volume of T2-hyperintense and T1-hypointense lesions - which are considered expression of MS inflammatory burden and axonal loss, respectively - did not differ significantly between fingolimod and interferon groups. A post hoc analysis of TRANSFORMS published 3 years later revealed that the benefit of fingolimod over interferon on relapse rate reduction was confirmed in several subgroups with the exception of male patients, subjects over 40 years of age, and patients with baseline EDSS score $>3.5 .{ }^{18}$ In the latter category also, the number of gadolinium-enhancing and new T2 lesions on brain MRI at 1 year did not differ significantly between fingolimod and interferon, while brain volume change remained in favor of fingolimod.

More recently, FREEDOMS II, a third Phase III trial of fingolimod, was conducted predominantly in USA and Canada. This was a double-blind randomized placebocontrolled 2-year study that included over 1,000 relapsingremitting MS patients, with a dropout rate of $28 \% .{ }^{19}$ Starting from November 2010 after fingolimod approval by the FDA, the study continued open-label to allow patients on placebo to switch to fingolimod. The trial replicated the findings of FREEDOMS regarding the ARR and MRI outcomes. However, the significant effect on reducing EDSS score progression observed in FREEDOMS was not confirmed in the FREEDOMS II trial, although it has to be noted that confirmed disability worsening occurred in a relatively low proportion of cases in both fingolimod and placebo groups (13.8\% and $17.8 \%$ at 2 years, respectively). On the other hand, disability as measured by MSFC showed a statistically significant change in favor of fingolimod treatment. In addition to "hard" outcome measures, FREEDOMS II included evaluation of patient-reported outcomes and symptoms using the Euro quality of life scale, Patient Reported Indices in Multiple Sclerosis, and Modified Fatigue Impact Scale. The scores obtained by patients in these questionnaires did not significantly differ between fingolimod and placebo groups.

Finally, the recently completed INFORMS trial showed no significant benefit of fingolimod on neurological disability in primary progressive MS patients treated for at least 3 years. $^{20}$

\section{Observational studies}

Extension studies conducted after the conclusion of randomized double-blind trials showed substantial clinical and MRI stability of MS patients continuing to receive fingolimod and overall amelioration of MS activity measures in patients switching from placebo or intramuscular interferon to fingolimod. ${ }^{21-23}$ However, this type of study is not designed to assess efficacy of a therapeutic intervention, and any conclusion in this regard based on observational open-label data should be avoided.

In the last 3 years, clinicians have devoted much attention to the matter of comparative efficacy of fingolimod versus other DMDs, particularly natalizumab that is the major "competitor" in terms of indications and clinical use profile in MS. There are no randomized trials directly comparing natalizumab and fingolimod efficacy. The only available data derive from case series and observational studies, either on MS patients who shifted from natalizumab to fingolimod, generally but not always showing a disease reactivation after the switch, ${ }^{24-31}$ or on active MS cases despite first-line diseasemodifying treatment who were switched to fingolimod or natalizumab as second-line option, suggesting a possible superior efficacy of natalizumab on relapse rate and progression of disability. ${ }^{32-34}$ One single retrospective study reported analogous efficacy of the two drugs. ${ }^{35}$ Interestingly, it has been suggested that early fingolimod start after natalizumab cessation (ie, no later than the recommended natalizumab 3-month washout interval) reduces relapse risk compared to longer treatment discontinuation. ${ }^{36-41}$ This strategy has proved also effective on MRI outcomes in a recent randomized trial comparing different natalizumab washout intervals before switching to fingolimod, ${ }^{42}$ suggesting that timing of 
DMD change may be crucial in MS patients with a history of active disease. Notably, it has been reported that fingolimod withdrawal can also lead to rebound of MS activity, possibly with severe clinical and MRI features. ${ }^{43-47}$

\section{Fingolimod safety and tolerability AEs in clinical trials}

Pooled analysis of long-term safety data from Phase II/III studies showed that fingolimod $0.5 \mathrm{mg}$ resulted in transient and rarely symptomatic $(0.5 \%)$ bradycardia and second-degree atrioventricular block on treatment initiation, minor blood pressure increase, frequent (9\%) but generally asymptomatic liver enzyme elevations, mild blood cholesterol level increase, and macular edema (ME; 0.4\%). In addition, fingolimodtreated subjects had a mild decrease of forced expiratory volume in the first second, which was not associated with any clinically relevant event. In fact, the reduction in peripheral blood lymphocyte count induced by the drug was the most commonly observed undesired effect, although only a total lymphocyte count below 200 cells $/ \mathrm{mm}^{3}$ was considered an $\mathrm{AE}$ as per protocol. Overall, infection rates and malignancy numbers were comparable between treatment groups. The incidence of serious AEs and treatment discontinuations due to AEs was similar with fingolimod $0.5 \mathrm{mg}$ and placebo. ${ }^{48}$

\section{Cardiovascular AEs}

Pooled analysis of the first dose monitoring data from pivotal Phase III trials revealed that fingolimod induced a transient reduction in heart rate and atrioventricular conduction, resulting in symptomatic bradycardia or second-degree atrioventricular block in only $0.6 \%$ and $0.2 \%$ of patients taking the $0.5 \mathrm{mg}$ dose, respectively. ${ }^{49}$ An Italian open study on the safety and tolerability of fingolimod first dose in a large group of 906 patients showed that most $(95.2 \%)$ did not experience any AE after fingolimod administration. ${ }^{50}$ Cardiovascular AEs occurred in 18 patients and included bradycardia (1.3\%), firstand second-degree atrioventricular block $(0.1 \%$ and $0.2 \%$, respectively), palpitations, sinus arrhythmia, and ventricular premature beats $(0.1 \%$ each). No event required medical intervention, being self-limited. Extended cardiac monitoring beyond 6 hours was required in less than $5 \%$ of patients. Similar results were reported in a Phase IIIb multicenter study as well as in the postmarketing experience. ${ }^{51-53}$

\section{Infections}

Two fatal cases of infection - one disseminated varicellazoster virus (VZV) infection and one herpes simplex virus (HSV) encephalitis - were reported in the TRANSFORMS among MS patients receiving the higher fingolimod dose. ${ }^{3}$ Overall, an increased incidence of VZV infections in fingolimod versus placebo-treated patients was observed in pooled data from six Phase II and III trials and their extensions for about 11,915 patient-years of fingolimod treatment. ${ }^{54}$ In fingolimod-treated patients the incidence of VZV infections was nearly twice that in controls (11 versus 6 per 1,000 patient-years) and included 149 cases: 125 (84\%) with uncomplicated herpes zoster (HZ) involving 2 contiguous dermatomes or less, 10 (7\%) ophthalmic HZ, 2 cases of disseminated $\mathrm{HZ}(1 \%)$ and 11 cases involving bilateral $\mathrm{HZ}$ or $\mathrm{HZ}$ in more than 2 contiguous dermatomes (7\%). The biological reason underlying the increased frequency of VZV infections in patients taking fingolimod is probably correlated with its effects on CD8 T-cell's effectors functions.

\section{Macular edema}

ME with or without visual symptoms has been reported in $0.5 \%$ of patients exposed to fingolimod in FREEDOMS and TRANSFORMS trials, occurring predominantly in the first 3-4 months of therapy. ${ }^{55}$ The condition is caused by fluid accumulation in the central retina or macula and symptoms include decreased acuity and metamorphopsia, although ME is frequently asymptomatic. ME detection is based on fundus oculi examination, retinal optical coherence tomography, and intravenous fluorescein angiography if needed. An ophthalmological evaluation is recommended at 3-4 months after treatment initiation and at regular intervals thereafter. ${ }^{56}$ The risk of fingolimod-associated $\mathrm{ME}$ is increased in patients with diabetes mellitus or uveitis, and most cases of ME in the trials occurred in patients older than age 41 . The mechanism of ME development is likely related to effects of S1P pathway activation on vascular permeability. Fingolimodrelated $\mathrm{ME}$ tends to resolve spontaneously after treatment discontinuation.

\section{Postmarketing reported AEs}

As of November 2014, approximately 112,000 patients were treated with fingolimod worldwide, and there were 195,000 patient-years of drug exposure in both clinical trials and the postmarketing, ${ }^{57}$ which is the ideal setting to monitor and recognize the occurrence of therapy AEs.

\section{Sudden death}

In November 2011, a report of the sudden death in a hypertensive patient on calcium-channel blockers and $\beta$-blockers less than 24 hours following first-dose fingolimod prompted the FDA and EMA to review safety data on Gilenya ${ }^{\circledR}$. FDA could 
not conclude that fingolimod was related to unexplained death, but it remained concerned about the cardiovascular effects of the drug after the first dose. ${ }^{58}$ In January 2012, CHMP assessed the reports of 15 cases of sudden or unexplained death in patients treated with Gilenya ${ }^{\circledR} .{ }^{59}$ Most of the deaths and cardiovascular events had occurred in patients with a history of cardiovascular problems or in those taking other medicines. However, the data were not conclusive as to whether Gilenya ${ }^{\circledR}$ was the cause of the death. Both FDA and EMA recommended changes to the product information to strengthen the existing warnings and ensure close monitoring of all patients, advising doctors to perform electrocardiogram monitoring for 6 hours after taking the first dose, to consider the need for extended monitoring, as well as to exclude patients on medications that can cause cardiac rhythm abnormalities. With these risk-minimization measures in place, both FDA and EMA conclusions were that the benefits of Gilenya ${ }^{\circledR}$ outweigh the risks. A recent Italian study on 53 MS patients starting fingolimod showed that the exposure to the drug significantly reduced left ventricular systolic function at 6 months, compared to natalizumab, which did not induce any significant change in a control group of $25 \mathrm{MS}$ subjects. ${ }^{60}$

\section{Progressive multifocal leukoencephalopathy}

Progressive multifocal leukoencephalopathy (PML) is a rare, severe, and potentially fatal brain infection caused by the John Cunningham virus (JCV), a common virus that is harmless in most people but can cause PML in patients with immunodeficiency or in those taking immunosuppressive drugs. In August 2013, FDA announced the first case of a patient developing PML while treated with Gilenya ${ }^{\circledR}$, and after that an additional case was reported. The two PML cases could not be conclusively linked to fingolimod because prior to this drug the patients had been treated with natalizumab, a known cause of PML. ${ }^{61,62}$

In August 2015, Novartis notified the FDA that one more patient developed definite PML and one had probable PML while taking fingolimod. Neither patient had prior exposure to natalizumab or other immunosuppressants for MS or any other medical condition. Gilenya ${ }^{\circledR}$ was stopped in both patients. ${ }^{63}$ The probable PML case was that of a 49-year-old patient with a 5-year history of MS previously treated with Rebif ${ }^{\circledR}$ (interferon- $\beta 1$ a from Merck Biopharma, Darmstadt, Germany) for 10 months and then with Gilenya ${ }^{\circledR}$, in addition to short-term corticosteroids before and during fingolimod treatment, for approximately 4 years. On a routine brain MRI, new lesions considered atypical for MS and compatible with PML were detected. A cerebrospinal fluid (CSF) sample taken at that time was positive for JCV DNA, and the diagnosis of probable PML was consistent with diagnostic criteria outlined in the American Academy of Neurology consensus statement ${ }^{64}$ in the absence of clinical signs or symptoms specific of PML. The definite PML case was a 54-year-old patient who developed PML after taking Gilenya ${ }^{\circledR}$ for approximately 2.5 years. The patient had a 14-year history of MS and had previously been treated with interferon- $\beta 1 \mathrm{~b}$ for approximately 11 years and with mesalazine for ulcerative colitis for the last 4 years. The patient also had a history of colorectal cancer treated with chemotherapy and radiation treatment in the past. The patient was hospitalized with suspected PML after developing new symptoms, including walking instability, clumsiness, inattention, somnolence, and mental sluggishness. Subsequently, the diagnosis of PML in this patient was established on the basis of symptoms, characteristic MRI findings, and the detection of JCV DNA in the CSF.

\section{Other viral infections}

A study evaluating postmarketing data referring to about 54,000 patient-years of fingolimod use found a reported rate of HZ lower than that of clinical trials ( 7 per 1,000 patientyears) ${ }^{54}$ However, as a shrewd editorial from Tyler ${ }^{65}$ noted, lower rates of AEs occurring in postmarketing studies may result from incomplete data capture or several issues related to the FDA Adverse Events Reporting System (FAERS) (voluntary basis, variable diagnostic accuracy, publicity bias, etc). Among the recommendations for risk mitigation in patients initiating a therapy with fingolimod, one has to consider two doses of the live-attenuated VZV vaccine 4 weeks apart in those individuals without serologic evidence of immunity to the virus 1 month before starting fingolimod. If complicated $\mathrm{HZV}$ infection develops in a patient receiving fingolimod, treatment should be discontinued and the case promptly treated with intravenous acyclovir $(10 \mathrm{mg} / \mathrm{kg} 3$ times daily for 7-10 days), while cases with uncomplicated disease can likely remain on fingolimod and be treated with oral formulations of acyclovir. ${ }^{54}$

Cases of severe HSV encephalitis and of VZV encephalitis in an immunized patient have also been reported in the postmarketing. ${ }^{66,67}$

\section{Rare AEs}

A variety of rare AEs associated with fingolimod treatment have been reported as single case reports or small case series including but not limited to posterior reversible 
encephalopathy syndrome,${ }^{68}$ cryptococcal meningoencephalitis and disseminated cryptococcosis, ${ }^{69,70}$ Kaposi sarcoma, ${ }^{71}$ tumefactive demyelination, ${ }^{72-74}$ severe autoimmune hemolytic anemia, ${ }^{75}$ asthma deterioration, ${ }^{76}$ amenorrhea, ${ }^{77}$ peripheral vascular adverse effects, ${ }^{78}$ ecchymotic angioedema-like cutaneous lesions, ${ }^{79}$ reversible cerebral vasoconstriction syndrome,${ }^{80}$ lymphomatoid papulosis, ${ }^{81}$ and hemophagocytic syndrome (this rare disorder due to cytokine dysregulation has been reported in association to infection in two patients treated with fingolimod for 9 and 15 months, respectively, both with fatal outcome). ${ }^{82,83}$

\section{Pregnancy outcomes}

The assessment of pregnancy outcomes from Phase II, III, and IV clinical studies showed that of the 66 pregnancies during which in utero exposure to fingolimod occurred, 24 were electively terminated, and five were either lost to follow up or were ongoing. Three-quarters of the remaining 37 pregnancies resulted in live births, while nine resulted in spontaneous abortion. In total, five cases $(7.5 \%)$ of abnormal fetal development were recorded: in all these cases, fetal exposure to the drug took place in the first trimester of pregnancy. ${ }^{84}$ Given the risks of teratogenicity, women of childbearing potential should use effective contraception during fingolimod therapy and for 2 months after discontinuation. ${ }^{85} \mathrm{~A}$ fingolimod pregnancy registry has been established to record data on pregnancy outcomes.

\section{Tolerability}

Most patients tolerate fingolimod well at a once-a-day dosing oral therapy. Side effects often reported are fatigue, headache, malaise, back pain, and gastrointestinal discomfort, which however are persisting or lead to treatment discontinuation within 1-2 years only in a minority of cases (around 10\%-15\%), according to both clinical trials findings and observational studies..$^{2,3,19,86}$ In the recent EPOC study, patient satisfaction and quality of life with fingolimod appeared to be better than with first-line injectable DMDs (ie, interferon- $\beta$ and glatiramer acetate), although the most commonly reported AEs were more frequent in patients who switched to fingolimod than in those who remained on injectable DMDs (headache: $12 \%$ versus $3 \%$; fatigue: $12 \%$ versus $6 \%$ ). ${ }^{87}$

\section{Discussion}

When fingolimod entered clinical use in 2010, it added to the preexisting therapeutic options for MS as an innovative oral DMD capable of strongly reducing inflammatory activity of the disease and with a promise for potential neuroprotection, as indicated by the novel mechanism of action and the results of both preclinical and clinical studies. In fact, data from pivotal trials - FREEDOMS, TRANSFORMS, and FREEDOMS II consistently showed that fingolimod significantly reduces the relapse rate, suppresses inflammatory activity on brain MRI, and slows brain atrophy progression in MS patients compared to placebo and intramuscular interferon. ${ }^{2,3,19}$ However, a reduction in neurological disability worsening was observed only in FREEDOMS, suggesting a potential heterogeneity of biological and clinical characteristics of MS populations across trials and also indicating the need for caution when interpreting the presence of a beneficial effect on brain atrophy that is dissociated from the effect on disability progression in MS. This is a well-known phenomenon in the history of MS DMDs trials, which could be explained by the fact that slowing of brain atrophy over 1-2 years may have marginal clinical impact in the short term of disease course, although a pooled analysis of data from 13 randomized controlled trials of various DMDs including $>13,500 \mathrm{MS}$ patients revealed a significant correlation between the effect on brain atrophy and the effect on disability progression over 2 years. ${ }^{88}$ Other possible explanations exist that are beyond the scope of this review. ${ }^{89}$

Unfortunately, no head-to-head randomized double-blind trials have been done to compare fingolimod to other established first- or second-line DMDs that are known to be more effective than intramuscular interferon once a week, such as subcutaneous interferon three times a week, mitoxantrone, or natalizumab. ${ }^{90}$ Nevertheless, with the limitations of indirect comparisons, it can be stated that the ARR reduction obtained with fingolimod versus placebo at 2 years (around 50\%), is greater than that observed with interferons, glatiramer acetate, or teriflunomide (around 30\%) and similar to that reported with dimethyl fumarate, among first-line DMDs, which share the same lacking or conflicting evidence for disability progression. ${ }^{91}$ Fingolimod trials have shown a lower ARR reduction compared to second-line therapies, such as natalizumab ( $-68 \%$ versus placebo), mitoxantrone $(-65 \%$ versus placebo), and the newly approved monoclonal antibody alemtuzumab (up to $-55 \%$ versus high dose interferon$\beta 1 \mathrm{a})$, as confirmed by a recent network meta-analysis. ${ }^{92}$ In addition, natalizumab, mitoxantrone, and alemtuzumab treatment are associated with a significant, although modest, reduction of disability progression risk, with the exception of the CARE-MS I trial in which the difference between alemtuzumab and interferon three times a week was not significant. In terms of MRI outcomes, fingolimod has an efficacy profile concerning inflammatory activity measures 
that is closer to first-line than second-line therapies, while it shows the more robust and consistent benefit on brain atrophy than all other DMDs. Fingolimod appears as an ideal second-line DMD for MS patients who are also eligible for natalizumab but test positive for serum anti-JCV antibodies (around 50\% of population) and are therefore at increased risk of PML during natalizumab treatment.

In 2010, FDA approved fingolimod for relapsing-remitting MS with or without previous use of interferon- $\beta$, while in 2011 EMA authorized the use of fingolimod as second-line treatment for relapsing-remitting MS with either high disease activity despite interferon treatment (extended to any other DMD in 2014) or untreated rapidly evolving severe disease as defined by clinical and MRI activity criteria. In 2012, the National Institute for health and Care Excellence recommended the use of the drug in the United Kingdom with an indication similar to that issued by EMA. One of the critical factors that led to fingolimod approval as a second-line DMD stood in safety data of Phase II and III trials, reinforced by postmarketing reports issued after Gilenya ${ }^{\circledR}$ marketing in USA as described in the "Fingolimod safety and tolerability" section of this review. Although fingolimod launch as the first new-generation "pill" for the treatment of MS stressed the advantage of an easy-to-use daily oral therapy, safety concerns were evident since the first trials and still remain to be vigilantly considered in each patient who is prescribed this drug. Potentially serious or even lethal adverse events, such as cardiovascular, hepatic, and infectious complications, may be prevented or identified in time by strict monitoring of the first dose and continuing to do so regularly during treatment period. However, an even more important step in assuring patient safety is the identification of comorbidities and other factors that predispose to certain types of adverse events prior to fingolimod initiation to minimize the risks versus the potential benefits. Fingolimod is contraindicated in MS cases with clinically relevant heart disease, infection, cancer, immunodeficiency, severe liver disease, uncontrolled hypertension, diabetes, lung disease, retinal conditions or a history of uveitis. Treatment with $\beta$-blockers and calcium channel blockers increases the risk of fingolimod-associated cardiac AEs, and so concomitant use of these drugs should be avoided. Simultaneous administration of other immunosuppressive or immunomodulatory therapies and long-standing steroid treatment are contraindicated because they may increase the risk of infections; short-term high-dose intravenous steroids typically used for MS relapses are allowed, although they must be prescribed with caution since some infectious serious AEs have been reported after exposure to high-dose methylprednisolone in patients receiving fingolimod. ${ }^{3}$ Finally, in case of diagnostic uncertainty, extreme caution has to be used before prescribing fingolimod since it could worsen certain MS-like conditions, such as neuromyelitis optica. ${ }^{93}$

\section{Conclusion}

Fingolimod is an effective oral treatment for reducing relapse rate, brain MRI inflammation, and brain atrophy in patients with relapsing-remitting MS naïve to treatment or previously treated with a first-line DMD. Fingolimod efficacy in preventing disability progression - as measured by EDSS score worsening - is uncertain in the short-term (1-2 years) and unknown in the medium long term (3 years or more), although the robust and consistent beneficial effect observed on brain atrophy may indicate a possible neuroprotective effect that is not captured by an empirical tool as the EDSS. Fingolimod tolerability is generally good or excellent. However, serious adverse events such as cardiac arrhythmias, macular edema, viral infections, severe lymphopenia, and other rare conditions may occur. For this reason, fingolimod appears a valid option for the treatment of patients with active relapsingremitting MS, after careful evaluation of concomitant conditions and medication and assuring adequate first-dose and long-term monitoring, as pointed out by the recently revised EMA report on Gilenya ${ }^{\circledR} .{ }^{94}$ Head-to-head clinical trials against natalizumab and other newer DMDs as well as observational studies to assess fingolimod safety in the long-term are warranted.

\section{Disclosure}

The authors report no conflicts of interest in this work.

\section{References}

1. Dutta R, Trapp BD. Relapsing and progressive forms of multiple sclerosis: insights from pathology. Curr Opin Neurol. 2014;27(3):271-278.

2. Kappos L, Radue EW, O'Connor P, et al. A placebo-controlled trial of oral fingolimod in relapsing multiple sclerosis. N Engl J Med. 2010;362(5): 387-401.

3. Cohen JA, Barkhof F, Comi G, et al. Oral fingolimod or intramuscular interferon for relapsing multiple sclerosis. N Engl J Med. 2010;362(5): 402-415.

4. Berkeley MJ. Fungi. Flora Novae-Zelandiae. Part II. Flowerless plants. In: Hooker JD, editor. The Botany of the Antarctic Voyage II. London, England: Lovell Reeve; 1855;172-210.

5. Lloyd CG. Isaria sinclairii. Mycol Writings. 1923;7:1179.

6. Paterson RR. Cordyceps: traditional Chinese medicine and another fungal therapeutic biofactory? Phytochemistry. 2008;69(7):1469-1495.

7. Fujita T, Inoue K, Yamamoto S, et al. Fungal metabolites. Part 11. A potent immunosuppressive activity found in Isaria sinclairii metabolite. J Antibiot. 1994;47(2):208-215.

8. Adachi K, Kohara T, Nakao N, et al. Design, synthesis, and structureactivity relationships of 2-substituted-2-amino-1,3-propanediols. Discovery of a novel immunosuppressant, FTY720. Bioorg Med Chem Lett. 1995;5(8):853-856. 
9. Tedesco-Silva H, Pescovitz MD, Cibrik D, et al. Randomized controlled trial of FTY720 versus MMF in de novo renal transplantation. Transplantation. 2006;82(12):1689-1697.

10. Salvadori M, Budde K, Charpentier B, et al. FTY720 versus MMF with cyclosporine in de novo renal transplantation: a 1-year, randomized controlled trial in Europe and Australasia. Am J Transplant. 2006;6(12): 2912-2921.

11. Webb M, Tham CS, Lin FF, et al. Sphingosine 1-phosphate receptor agonists attenuate relapsing-remitting experimental autoimmune encephalitis in SJL mice. J Neuroimmunol. 2004;153:108-121.

12. Kappos L, Antel J, Comi G, et al. Oral fingolimod (FTY720) for relapsing multiple sclerosis. $N$ Engl J Med. 2006;355:1124-1140.

13. Cleveland clinic. Cleveland clinic innovations. Available from: http:// innovations.clevelandclinic.org/Summit-(1)/Top-10-Innovations/ Top-10-for-2011.aspx\#.VeIITM65dLw. Accessed September 24, 2015.

14. Landers P. MS drug's epic journey from folklore to lab. The Wall Street Journal. June 22, 2010. Available from: http://www.wsj.com/articles/ SB10001424052748704256304575320714138159240. Accessed September 25, 2015.

15. Chun J, Hartung HP. Mechanism of action of oral fingolimod (FTY720) in multiple sclerosis. Clin Neuropharmacol. 2010;33(2):91-101.

16. Brinkmann V, Billich A, Baumruker T, et al. Fingolimod (FTY720): discovery and development of an oral drug to treat multiple sclerosis. Nat Rev Drug Discov. 2010;9(11):883-897.

17. Devonshire V, Havrdova E, Radue EW, et al. Relapse and disability outcomes in patients with multiple sclerosis treated with fingolimod: subgroup analyses of the double-blind, randomised, placebo-controlled FREEDOMS study. Lancet Neurol. 2012;11(5):420-428.

18. Cohen JA, Barkhof F, Comi G, et al. Fingolimod versus intramuscular interferon in patient subgroups from TRANSFORMS. J Neurol. 2013; 260(8):2023-2032.

19. Calabresi PA, Radue EW, Goodin D, et al. Safety and efficacy of fingolimod in patients with relapsing-remitting multiple sclerosis (FREEDOMS II): a double-blind, randomised, placebo-controlled, phase 3 trial. Lancet Neurol. 2014;13(6):545-556.

20. Novartis. Novartis provides update on fingolimod Phase III trial in primary progressive MS (PPMS). Available from: https://www.novartis. com/news/media-releases/novartis-provides-update-fingolimod-phaseiii-trial-primary-progressive-ms-ppms. Accessed September 20, 2015.

21. Kappos L, O'Connor P, Radue EW, et al. Long-term effects of fingolimod in multiple sclerosis: the randomized FREEDOMS extension trial. Neurology. 2015;84(15):1582-1591.

22. Cohen JA, Khatri B, Barkhof F, et al. Long-term (up to 4.5 years) treatment with fingolimod in multiple sclerosis: results from the extension of the randomised TRANSFORMS study. J Neurol Neurosurg Psychiatry. Epub 2015 Jun 25.

23. Montalban X, Comi G, Antel J, et al. Long-term results from a phase 2 extension study of fingolimod at high and approved dose in relapsing multiple sclerosis. J Neurol. Epub 2015 Sep 4.

24. Centonze D, Rossi S, Rinaldi F, Gallo P. Severe relapses under fingolimod treatment prescribed after natalizumab. Neurology. 2012;79: 2004-2005.

25. Rinaldi F, Seppi D, Calabrese M, Perini P, Gallo P. Switching therapy from natalizumab to fingolimod in relapsing-remitting multiple sclerosis: clinical and magnetic resonance imaging findings. Mult Scler. 2012;18:1640-1643.

26. Daelman L, Maitrot A, Maarouf A, Chaunu MP, Papeix C, Tourbah A. Severe multiple sclerosis reactivation under fingolimod 3 months after natalizumab withdrawal. Mult Scler. 2012;18:1647-1649.

27. Jander S, Turowski B, Kieseier BC, Hartung HP. Emerging tumefactive multiple sclerosis after switching therapy from natalizumab to fingolimod. Mult Scler. 2012;18:1650-1652.

28. Sempere AP, Martín-Medina P, Berenguer-Ruiz L, et al. Switching from natalizumab to fingolimod: an observational study. Acta Neurol Scand. 2013;128:e6-e10.
29. Baldi E, Guareschi A, Vitetta F, et al. Previous treatment influences fingolimod efficacy in relapsing-remitting multiple sclerosis: results from an observational study. Curr Med Res Opin. 2014;30(9):1849-1855.

30. Hoepner R, Havla J, Eienbröker C, et al. Predictors for multiple sclerosis relapses after switching from natalizumab to fingolimod. Mult Scler. 2014;20(13):1714-1720.

31. Bianco A, Patanella AK, Nociti V, et al. Second-line therapy with fingolimod for relapsing-remitting multiple sclerosis in clinical practice: the effect of previous exposure to natalizumab. Eur Neurol. 2015;73(1-2):57-65.

32. Gajofatto A, Bianchi MR, Deotto L, Benedetti MD. Are natalizumab and fingolimod analogous second-line options for the treatment of relapsing-remitting multiple sclerosis? A clinical practice observational study. Eur Neurol. 2014;72(3-4):173-180.

33. Carruthers RL, Rotstein DL, Healy BC, Chitnis T, Weiner HL, Buckle GJ. An observational comparison of natalizumab vs fingolimod using JCV serology to determine therapy. Mult Scler. 2014;20(10):1381-1390.

34. Kalincik T, Horakova D, Spelman T, et al. Switch to natalizumab versus fingolimod in active relapsing-remitting multiple sclerosis. Ann Neurol. 2015;77(3):425-435.

35. Braune S, Lang M, Bergmann A; NTC Study Group. Second line use of fingolimod is as effective as natalizumab in a German out-patient RRMS cohort. J Neurol. 2013;260:2981-2985.

36. Laroni A, Brogi D, Milesi V, Abate L, Uccelli A, Mancardi G. Early switch to fingolimod may decrease the risk of disease recurrence after natalizumab interruption. Mult Scler. 2012;19:1236-1237.

37. Havla J, Tackenberg B, Hellwig K, et al. Fingolimod reduces recurrence of disease activity after natalizumab withdrawal in multiple sclerosis. J Neurol. 2013;260:1382-1387.

38. de Seze J, Ongagna JC, Collongues N, et al. Reduction of the washout time between natalizumab and fingolimod. Mult Scler. 2013;19(9):1248.

39. Cohen M, Maillart E, Tourbah A, et al. Switching from natalizumab to fingolimod in multiple sclerosis: a French prospective study. JAMA Neurol. 2014;71(4):436-441.

40. Comi G, Gold R, Dahlke F, et al. Relapses in patients treated with fingolimod after previous exposure to natalizumab. Mult Scler. 2015;21(6): 786-790.

41. Iaffaldano P, Lucisano G, Pozzilli C, et al. Fingolimod versus interferon beta/glatiramer acetate after natalizumab suspension in multiple sclerosis. Brain. 2015;138(Pt 11):3275-3286.

42. Kappos L, Radue EW, Comi G, et al. Switching from natalizumab to fingolimod: a randomized, placebo-controlled study in RRMS. Neurology. 2015;85(1):29-39.

43. Alroughani R, Almulla A, Lamdhade S, Thussu A. Multiple sclerosis reactivation postfingolimod cessation: is it IRIS? BMJ Case Rep. 2014;2014. pii: bcr2014206314.

44. La Mantia L, Prone V, Marazzi MR, Erminio C, Protti A. Multiple sclerosis rebound after fingolimod discontinuation for lymphopenia. Neurol Sci. 2014;35(9):1485-1486.

45. Ghezzi A, Rocca MA, Baroncini D, et al. Disease reactivation after fingolimod discontinuation in two multiple sclerosis patients. J Neurol. 2013;260(1):327-329.

46. Sempere AP, Berenguer-Ruiz L, Feliu-Rey E. Rebound of disease activity during pregnancy after withdrawal of fingolimod. Eur J Neurol. 2013;20(8):e109-e110.

47. Piscolla E, Hakiki B, Pastò L, Razzolini L, Portaccio E, Amato MP. Rebound after fingolimod suspension in a pediatric-onset multiple sclerosis patient. J Neurol. 2013;260(6):1675-1677.

48. Kappos L, Cohen J, Collins W, et al. Fingolimod in relapsing multiple sclerosis: an integrated analysis of safety findings. Mult Scler Relat Disord. 2014;3(4):494-504.

49. DiMarco JP, O'Connor P, Cohen JA, et al. First-dose effects of fingolimod: pooled safety data from three phase 3 studies. Mult Scler Relat Disord. 2014;3(5):629-638.

50. Laroni A, Brogi D, Morra VB, et al. Safety of the first dose of fingolimod for multiple sclerosis: results of an open-label clinical trial. $B M C$ Neurol. 2014;14:65. 
51. Gold R, Comi G, Palace J, et al. Assessment of cardiac safety during fingolimod treatment initiation in a real-world relapsing multiple sclerosis population: a phase 3b, open-label study. J Neurol. 2014;261(2): 267-276.

52. Ramseier SP, Roth S, Czaplinski A. A Swiss real world best practice experience in three different clinical settings of the 6 hour fingolimod first dose observation procedure. BMC Pharmacol Toxicol. 2015;16:7.

53. Paolicelli D, Manni A, Direnzo V, et al. Long-term cardiac safety and tolerability of fingolimod in multiple sclerosis: a postmarketing study. J Clin Pharmacol. 2015;55(10):1131-1136.

54. Arvin AM, Wolinsky JS, Kappos L, et al. Varicella-zoster virus infections in patients treated with fingolimod: risk assessment and consensus recommendations for management. JAMA Neurol. 2015;72(1):31-39.

55. Jain N, Bhatti MT. Fingolimod-associated macular edema: incidence, detection, and management. Neurology. 2012;78(9):672-680.

56. Zarbin MA, Jampol LM, Jager RD, et al. Ophthalmic evaluations in clinical studies of fingolimod (FTY720) in multiple sclerosis. Ophthalmology. 2013;120(7):1432-1439.

57. gilenyaworldwatch.com [homepage on the Internet]. Available from: http:// www.gilenyaworldwatch.com/English.html. Accessed September 25, 2015.

58. US Food and Drug Administration. FDA Drug Safety Communication: revised recommendations for cardiovascular monitoring and use of multiple sclerosis drug Gilenya (fingolimod). Available from: http://www. fda.gov/Drugs/DrugSafety/ucm303192.htm. Accessed September 25, 2015 .

59. European Medicines Agency. European Medicines Agency gives new advice to better manage risk of adverse effects on the heart with Gilenya. Available from: http://www.ema.europa.eu/docs/en_GB/ document_library/Press_release/2012/04/WC500125690.pdf. Accessed November 11, 2015.

60. Racca V, Di Rienzo M, Cavarretta R, et al. Fingolimod effects on left ventricular function in multiple sclerosis. Mult Scler. Epub 2015 Jun 3 .

61. Killestein J, Vennegoor A, van Golde AE, Bourez RL, Wijlens ML, Wattjes MP. PML-IRIS during fingolimod diagnosed after natalizumab discontinuation. Case Rep Neurol Med. 2014;2014:307872.

62. Calic Z, Cappelen-Smith C, Hodgkinson SJ, McDougall A, Cuganesan R, Brew BJ. Treatment of progressive multifocal leukoencephalopathy-immune reconstitution inflammatory syndrome with intravenous immunoglobulin in a patient with multiple sclerosis treated with fingolimod after discontinuation of natalizumab. $J$ Clin Neurosci. 2015;22(3):598-600.

63. US Food and Drug Administration. FDA Drug Safety Communication: FDA warns about cases of rare brain infection with MS drug Gilenya (fingolimod) in two patients with no prior exposure to immunosuppressant drugs. Available from: http://www.fda.gov/Drugs/DrugSafety/ ucm456919.htm. Accessed September 25, 2015.

64. Berger JR, Aksamit AJ, Clifford DB, et al. PML diagnostic criteria: consensus statement from the AAN Neuroinfectious Disease Section. Neurology. 2013;80(15):1430-1438.

65. Tyler KL. Fingolimod and risk of varicella-zoster virus infection: back to the future with an old infection and a new drug. JAMA Neurol. 2015;72(1):10-13.

66. Pfender N, Jelcic I, Linnebank M, Schwarz U, Martin R. Reactivation of herpesvirus under fingolimod: a case of severe herpes simplex encephalitis. Neurology. 2015;84(23):2377-2378.

67. Issa NP, Hentati A. VZV encephalitis that developed in an immunized patient during fingolimod therapy. Neurology. 2015;84(1):99-100.

68. Lindå $\mathrm{H}$, von Heijne A. A case of posterior reversible encephalopathy syndrome associated with Gilenya ${ }^{\circledR}$ (fingolimod) treatment for multiple sclerosis. Front Neurol. 2015;6:39.

69. Achtnichts L, Obreja O, Conen A, Fux CA, Nedeltchev K. Cryptococcal meningoencephalitis in a patient with multiple sclerosis treated with fingolimod. JAMA Neurol. 2015;72(10):1203-1205.

70. Huang D. Disseminated cryptococcosis in a patient with multiple sclerosis treated with fingolimod. Neurology. 2015;85(11):1001-1003.
71. Tully T, Barkley A, Silber E. Kaposi sarcoma in a patient with relapsing-remitting multiple sclerosis receiving fingolimod. Neurology. 2015;84(19):1999-2001.

72. Pilz G, Harrer A, Wipfler P, et al. Tumefactive MS lesions under fingolimod: a case report and literature review. Neurology. 2013;81(19): 1654-1658.

73. Hellmann MA, Lev N, Lotan I, et al. Tumefactive demyelination and a malignant course in an MS patient during and following fingolimod therapy. J Neurol Sci. 2014;344(1-2):193-197.

74. Harirchian MH, Taalimi A, Siroos B. Emerging tumefactive MS after switching therapy from interferon-beta to fingolimod: a case report. Mult Scler Relat Disord. 2015;4(5):400-402.

75. Lysandropoulos AP, Benghiat F. Severe auto-immune hemolytic anemia in a fingolimod-treated multiple sclerosis patient. Mult Scler. 2013;19(11):1551-1552.

76. van Rossum JA, Looysen EE, Daniels JM, Killestein J. Fingolimodinduced asthma deterioration in a patient with relapsing-remitting multiple sclerosis. Mult Scler. 2014;20(13):1792-1793.

77. Alroughani R. Fingolimod-associated amenorrhea: a report of three cases. Mult Scler. 2014;20(12):1662-1664.

78. Russo M, Guarneri C, Mazzon E, Sessa E, Bramanti P, Calabrò RS. Fingolimod-associated peripheral vascular adverse effects. Mayo Clin Proc. 2015;90(10):1424-1427.

79. Masera S, Chiavazza C, Mattioda A, et al. Occurrence of ecchymotic angioedema-like cutaneous lesions as a possible side effect of fingolimod. Mult Scler. 2014;20(12):1666-1667.

80. Kraemer M, Weber R, Herold M, Berlit P. Reversible cerebral vasoconstriction syndrome associated with fingolimod treatment in relapsingremitting multiple sclerosis three months after childbirth. Mult Scler. 2015;21(11):1473-1475.

81. Samaraweera AP, Cohen SN, Akay EM, Evangelou N. Lymphomatoid papulosis: a cutaneous lymphoproliferative disorder in a patient on fingolimod for multiple sclerosis. Mult Scler. Epub 2015 Jul 28.

82. Abreu P, Peixoto C, Carvalho C, Santos L, Sarmento A, Sá MJ. A case of hemophagocytic lymphohistiocytosis syndrome in a patient with multiple sclerosis on fingolimod therapy [abstract]. Neurology. 2014;82(Suppl 10):P2.206.

83. European Medicines Agency. Gilenya: procedural steps taken and scientific information after the authorisation. Available from: http://www.ema. europa.eu/docs/en_GB/document_library/EPAR_-_Procedural_steps_ taken_and_scientific_information_after_authorisation/human/002202/ WC500111236.pdf. Accessed September 25, 2015.

84. Karlsson G, Francis G, Koren G, et al. Pregnancy outcomes in the clinical development program of fingolimod in multiple sclerosis. Neurology. 2014;82(8):674-680.

85 . Jones B. Multiple sclerosis: study reinforces need for contraception in women taking fingolimod. Nat Rev Neurol. 2014;10(3):125.

86. Hersh CM, Hara-Cleaver C, Rudick RA, Cohen JA, Bermel RA, Ontaneda D. Experience with fingolimod in clinical practice. Int $J$ Neurosci. 2015;125(9):678-685.

87. Fox E, Edwards K, Burch G, et al. Outcomes of switching directly to oral fingolimod from injectable therapies: results of the randomized, open-label, multicenter, Evaluate Patient OutComes (EPOC) study in relapsing multiple sclerosis. Mult Scler Relat Disord. 2014;3(5):607-619.

88. Sormani MP, Arnold DL, De Stefano N. Treatment effect on brain atrophy correlates with treatment effect on disability in multiple sclerosis. Ann Neurol. 2014;75(1):43-49.

89. Calabrese M, Gajofatto A, Benedetti MD. Therapeutic strategies for relapsing-remitting multiple sclerosis: a special focus on reduction of grey matter damage as measured by brain atrophy. Expert Rev Neurother. 2014;14(12):1417-1428.

90. Filippini G, Del Giovane C, Vacchi L, et al. Immunomodulators and immunosuppressants for multiple sclerosis: a network meta-analysis. Cochrane Database Syst Rev. 2013;6:CD008933.

91. Gajofatto A, Benedetti MD. Treatment strategies for multiple sclerosis: when to start, when to change, when to stop? World J Clin Cases. 2015;3(7):545-555. 
92. Tramacere I, Del Giovane C, Salanti G, D’Amico R, Filippini G. Immunomodulators and immunosuppressants for relapsing-remitting multiple sclerosis: a network meta-analysis. Cochrane Database Syst Rev. 2015;9:CD011381.

93. Papadopoulos MC, Bennett JL, Verkman AS. Treatment of neuromyelitis optica: state-of-the-art and emerging therapies. Nat Rev Neurol. 2014;10(9):493-506.
94. European Medicines Agency. Gilenya. Available from: http://www.ema. europa.eu/ema/index.jsp?curl=pages/medicines/human/medicines/002202/ human_med_001433.jsp\&mid=WC0b01ac058001d124. Accessed November 11, 2015.

Drug, Healthcare and Patient Safety

\section{Publish your work in this journal}

Drug, Healthcare and Patient Safety is an international, peer-reviewed open-access journal exploring patient safety issues in the healthcare continuum from diagnostic and screening interventions through to treatment, drug therapy and surgery. The journal is characterized by the rapic reporting of reviews, original research, clinical, epidemiological and
Dovepress

post-marketing surveillance studies, risk management, health literacy and educational programs across all areas of healthcare delivery. The manuscript management system is completely online and includes a very quick and fair peer-review system. Visit http://www.dovepress.com/ testimonials.php to read real quotes from published authors.

Submit your manuscript here: http://www.dovepress.com/drug-healthcare-and-patient-safety-journal 